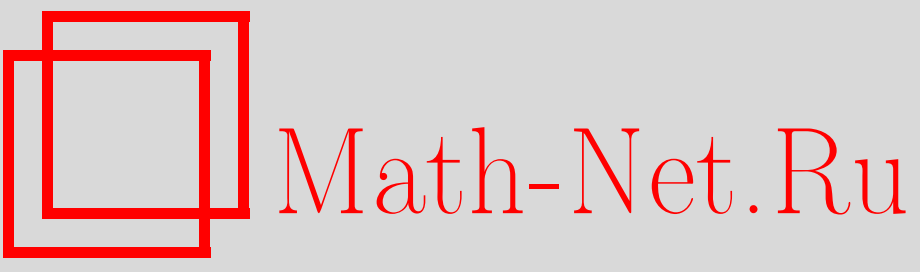

А. В. Карташова, Решетки топологий унарных алгебр, УМH, 2000, том 55, выпуск $6,141-142$

DOI: https://doi.org/10.4213/rm345

Использование Общероссийского математического портала Math-Net.Ru подразумевает, что вы прочитали и согласны с пользовательским соглашением

http://www.mathnet.ru/rus/agreement

Параметры загрузки:

IP: 3.85 .7 .115

26 апреля 2023 г., 17:02:07 


\title{
РЕШЕТКИ ТОПОЛОГИЙ УНАРНЫХ АЛГЕБР
}

\author{
A. B. КАРТАШОВА
}

Пусть $\mathfrak{A}=\langle A, \Omega\rangle$ - произвольная универсальная алгебра. Топология на множестве $A$, относительно которой каждая операция из $\Omega$ непрерывна, назьвается топологией на алгебре $\mathfrak{A}$. Известно [1], что топологии на алгебре $\mathfrak{A}$ образуют полную решетку по включению. Будем назьвать эту решетку решеткой топологий алгебры $\mathfrak{A}$ и обозначать через $\Im(\mathfrak{A})$.

Tеорема 1. Решетка $\widetilde{\operatorname{Con}} \mathfrak{A}$, двойственная к решетке Con $\mathfrak{A}$ конгруәниий произвольной алгебры $\mathfrak{A}=\langle A, \Omega\rangle$, изоморфно вложима в решетку $\Im(\mathfrak{A})$ ее топологий.

Для любой конгруэнции $\theta$ алгебры $\mathfrak{A}$ через $\tau(\theta)$ обозначим топологию, базой которой служат классы данной конгруэнции.

ТеОрема 2. Пусть $\varphi: \mathfrak{A} \rightarrow \mathfrak{B}$ - произвольныц эпиморфизм алгебр. Тогда решет$\kappa a \Im(\mathfrak{B})$ топологий алгебры $\mathfrak{B}$ изоморфна главному идеалу решетки $\Im(\mathfrak{A})$, порожденному топологией $\tau(\theta)$, где $\theta$ - ядро эпиморфизма $\varphi$.

В общем случае решетка $\Im(\mathfrak{A})$ топологий алгебры $\mathfrak{A}=\langle A, \Omega\rangle$ не является подрешеткой решетки по включению всех топологий на множестве $A$ (см. [2]). Однако простая проверка показывает, что если сигнатура $\Omega$ состоит только из унарных символов, то $\Im(A)$ - полная подрешетка решетки топологий на множестве $A$.

Теорема 3. Пусть $\mathfrak{A}$ - произвольная унарная алгебра и $\mathfrak{B}$ - ее подалгебра. Тогда решетка $\Im(\mathfrak{B})$ вложима в качестве главного фильтра в решетку $\Im(\mathfrak{A})$.

Для алгебр произвольной сигнатуры утверждение теоремы 3 не всегда справедливо.

Теорема 4. Пусть $\Omega$ - произвольная функциональная сигнатура, содержащая хотя бы один символ, арность которого больше 1. Тогда существует трехэлементная алгебра $\mathfrak{A}$ сигнатурь $\Omega$ такал, что решетка топологий алгебры $\mathfrak{A}$ двухәлементна, а решетка топологий некоторой подалгебры этой алгебры четырехэлементна.

Алгебра $\mathfrak{A}=\langle A, f\rangle$ с одной унарной операцией $f$ называется унаром.

ПреДЛОЖенИЕ 5 . Пусть $\mathfrak{A}-$ произвольный унар. Тогда

1) решетка $(\mathfrak{A})$ не может бить счетной;

2) если мнохество А несчетно, то $|\Im(\mathfrak{A})|=2^{2^{|\mathfrak{A}|}}$.

Подунар $(a)$, порожденный элементом $a$ произвольного унара $\mathfrak{A}=\langle A, f\rangle$, называется циклом длины $n \geqslant 1$, если $f^{n}(a)=a$ и $f^{k}(a) \neq a$ при $0<k<n$. При этом элемент $a$ называется циклическим.

СЛЕДСТВИЕ 6. Пусть $\mathfrak{A}$ - произвольньй унар. Тогда решетка $\Im(\mathfrak{A})$ изоморфна решетке Cоп $\mathfrak{A}$ в том и только том случае, когда $\mathfrak{A}$ является ииклом.

СледствиЕ 7. Справедливы следующие утверждения:

1) существуют такие унары $\mathfrak{A}$ и $\mathfrak{B}$, что решетки Con $\mathfrak{A}$ и Con $\mathfrak{B}$ конгруәнций унаров $\mathfrak{A}$ и $\mathfrak{B}$ соответственно изоморфньи, а решетки $\Im(\mathfrak{A})$ и $\Im(\mathfrak{B})$ неизоморфньг;

2) существуют такие унары $\mathfrak{C}$ и $\mathfrak{D}$, что решетки $\Im(\mathfrak{C})$ и $\Im(\mathfrak{D})$ топологий этих унаров изоморфны, а решетки Con $\mathfrak{C}$ и Con $\mathfrak{D}$ не являются изоморфными.

Tеорема 8. Множество атомов в решетке $\Im(\mathfrak{A})$ топологий унара $\mathfrak{A}=\langle A, f\rangle$ конечно тогда и только тогда, когда $\mathfrak{A}$ содержит конечное число связнъх компонент, среди которых хотя бы одна имеет иикл, и число әлементов, не имеющих прообраза при отображсении $f$, конечно. В этом случае справедлива формула:

$$
\alpha_{\mathfrak{A}}=2^{\beta_{\mathfrak{A}}+1}+2^{\gamma_{\mathfrak{A}}}+\sum_{p \in L_{\mathfrak{A}}} p^{\gamma_{\mathfrak{A}}-1}-4
$$


где $\alpha_{\mathfrak{A}}-$ мощность множества атомов в решетке $\Im(\mathfrak{A})$ и $\beta_{\mathfrak{A}}-$ мощность множества әлементов, не имеющих прообраза при отображении $f, \gamma_{\mathfrak{A}}$-мощность множества связных компонент унара $\mathfrak{A}, L_{\mathfrak{A}}$ - множество всех простых чисел $p$, делящ,и длины циклов $\mathfrak{A}$.

Теорема 9. Справедливы следующие утверждения:

1) множество атомов в решетке $\Im(\mathfrak{A})$ произвольного унара $\mathfrak{A}$ счетно тогда и только тогда, когда $\beta_{\mathfrak{A}}<\aleph_{0}, \gamma_{\mathfrak{A}}<\aleph_{0}$ и унар $\mathfrak{A}$ не содержит ииклов;

2) множество атомов в решетке $((\mathfrak{A )}$ несчетно тогда и только тогда, когда либо $\beta_{\mathfrak{A}} \leqslant \aleph_{0}$, либо $\gamma_{\mathfrak{A}} \geqslant \aleph_{0}$, при этом

$$
\alpha_{\mathfrak{A}}=2^{\max \left\{\beta_{\mathfrak{A}}, \gamma_{\mathfrak{A}}\right\}} .
$$

$\left(\alpha_{\mathfrak{A}}, \beta_{\mathfrak{A}}, \gamma_{\mathfrak{A}}\right.$ имеют тот же смисл, что и в теореме 8.)

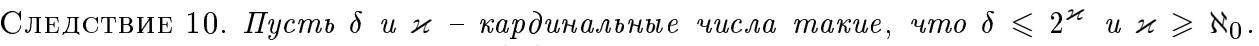
Тогда найдется такой унар $\mathfrak{A}$, что $|\mathfrak{A}|=\varkappa u \alpha_{\mathfrak{A}}=\delta$.

Из теорем 1-3 и результатов работы [3] следует

Теорема 11. Модулярная решетка изоморфна решетке топологий $(\mathfrak{A})$ некоторого унара $\mathfrak{A}$ тогда и только тогда, когда она изоморфна решетке делителей некоторого натурального числа $n$.

СледСтвиЕ 12. Если решетка $(\mathfrak{A})$ топологий произвольного унара $\mathfrak{A}$ модулярна, то она дистрибутивна.

СледСтвие 13. Решетка $(\mathfrak{A})$ топологий унара $\mathfrak{A}$ является цепью тогда и только тогда, когда $\mathfrak{A}-$ чикл длинь $p^{n}$, где $p$ - простое число, $n \in \mathbb{N} \cup\{0\}$.

В работе [4] показано, что решетка по включению всех топологий на произвольном множестве является решеткой с дополнениями. Обобщением этого факта является

Tеорема 14. Решетка $\Im(\mathfrak{A})$ топологий унара $\mathfrak{A}=\langle A, f\rangle$ является решеткой с дополнениями тогда и только тогда, когда для каждого әлемента а әлемент $f(a)$ является циклическим и длинь всех ииклов унара равнь некоторому фиксированному числу $n$, где $n$ свободно от квадратов.

Tеорема 15. Решетка $\Im(\mathfrak{A})$ mопологий унара $\mathfrak{A}$ является решеткой с псевдодополнениями тогда и только тогда, когда $\mathfrak{A}$ является либо однопорожденным конечным унаром, либо - обвединением двух ииклов, длины которых взаимно просты.

\section{СПИСОК ЛИТЕРАТУРЫ}

[1] Орлов С. Д. // Упорядоченные множества и решетки: Межвуз. науч. сб. №2. Саратов: Изд-во Саратов. ун-та, 1974. С. 68-71. [2] Arnautov V. I., Glavatsky S. T., Mikhalev A. V. Introduction to the Theory of Topological Rings and Modules. New York: Marcel Dekker, 1996. [3] Егорова Д. П. // Упорядоченные множества и решетки: Межвуз. науч. сб. № 5. Саратов: Изд-во Саратов. ун-та, 1978. С. 11-44. [4] Steiner A. K. // Trans. Amer. Math. Soc. 1966. V. 122. P. 379-398. 